\title{
Restos de Alvarezsauridae (Theropoda, Coelurosauria) en la Formación Allen (Campaniano-Maastrichtiano), en Salitral Ojo de Agua, Provincia de Río Negro, Argentina
}

\author{
Leonardo Salgado ${ }^{1}$, Rodolfo A. Coria ${ }^{2}$, Andrea B. Arcucci ${ }^{3}$, Luis M. Chiappe
}

\author{
' CONICET-INIBIOMA, Museo de Geología y Paleontología, Universidad Nacional del Comahue, Buenos Aires 1400, 8300 \\ Neuquén, Argentina. \\ lsalgado@uncoma.edu.ar \\ 2 CONICET, Museo 'Carmen Funes', 8318 Plaza Huincul, Neuquén, Argentina. \\ coriarod@copelnet.com.ar \\ 3 Universidad Nacional de San Luis, Facultad de Química, Bioquímica y Farmacia, Chacabuco y Pedernera 5700, San Luis, Argentina. \\ arcucci@satlink.com.ar \\ ${ }^{4}$ Natural History Museum of Los Angeles County, 900 Exposition Boulevard, Los Angeles, CA 90007, USA. \\ lchiappe@nhm.org
}

RESUMEN. Se presentan nuevos restos provenientes de estratos campaniano-maastrichtianos de la Formación Allen, en Salitral Ojo de Agua (Río Negro, Argentina), los cuales son asignables a Alvarezsauridae indet. Este clado de pequeños terópodos celurosaurios es conocido de estratos de edad similar en Mongolia y de sedimentitas más antiguas (de edad turoniana y coniaciana) en Argentina. De este modo, el material que aquí se informa extiende el registro temporal de los alvarezsáuridos del Hemisferio Sur hasta el Cretácico Tardío alto. Los huesos reportados estaban asociados a cáscaras de huevo de la oofamilia Elongatoolithidae, los cuales son actualmente atribuidos a Theropoda.

Palabras claves: Alvarezsauridae, Elongatoolithidae, Formación Allen, Río Negro, Argentina.

ABSTRACT. Remains of Alvarezsauridae (Theropoda, Coelurosauria) in the Allen Formation (CampanianMaastrichthian), in Salitral Ojo de Agua, Río Negro Province, Argentina. We present new remains from the Campanian-Maastrichtian beds of the Allen Formation, in Salitral Ojo de Agua (Río Negro, Argentina), which are assignable to Alvarezsauridae indet. This clade of small coelurosaurian theropods is known from strata of similar age in Mongolia, and from older sediments (Turonian and Coniacian in age) in Argentina. Thus, the material presented here extends the temporal record of the alvarezsaurids from the Southern Hemisphere up to the Latest Cretaceous. The fossil bones reported were associated to eggshells of the oofamily Elongatoolithidae, which are actually attributed to Theropoda. 


\section{Introducción}

Las rocas pertenecientes a unidades campaniano-maastrichtianas expuestas en el norte de la Patagonia Argentina son prolíficas en restos fósiles de tetrápodos continentales (Casamiquela, 1964; Bonaparte et al., 1984; Albino, 1986; Bonaparte, 1987; Powell, 1992; Salgado y Coria, 1996; Salgado y Azpilicueta, 2000; de la Fuente et al., 2001; Martinelli y Forasiepi, 2004; Coria, 2001; Coria y Salgado, 2005; Coria et al., 2007; Salgado et al., 2007a,b). La Formación Allen, cuyos afloramientos se hallan ampliamente distribuidos en el norte y centro de la provincia de Río Negro, es una de las unidades del final del Cretácico más importantes de Argentina ya sea por abundancia en restos fósiles como por diversidad de taxones registrados (véase Martinelli y Forasiepi, 2004). Sin embargo, la información sobre la exacta procedencia estratigráfica de los materiales recolectados, o incluso su ubicación geográfica precisa, es a menudo omitida en la literatura disponible. En relación con la Formación Allen, estos datos son fundamentales debido a la variedad de paleoambientes que comprende, desde continentales con restos de huesos y huevos de dinosaurios (Coria, 2001; Coria et al., 2007; Salgado et al., 2007b), hasta marino-litorales con restos de plesiosaurios (Salgado et al., 2007a).

En esta contribución se describen nuevos materiales óseos de dinosaurios alvarezsáuridos (Theropoda, Coelurosauria), de los cuales se tiene un adecuado control geográfico y estratigráfico. Sin duda, el conocimiento que aportan estos restos ayuda a una mejor comprensión de la evolución de los linajes de dinosaurios carnívoros cretácicos de la Patagonia, como así también extiende significativamente el registro temporal sudamericano de los alvarezsáuridos, considerados como el taxón hermano de las aves (Chiappe et al., 2002), o como un clado de Maniraptoriformes basales (Norell et al., 2001; Novas y Pol, 2002; Clark et al., 2002; Hwang et al., 2002; Makovicky et al., 2005; Göhlich y Chiappe, 2006). Los nombres que figuran en la sección 'Descripciones Sistemáticas' (Theropoda, Tetanurae, etc.) no corresponden a ninguna de las categorías de la taxonomía tradicional linneana; son simplemente nombres de clados, según el criterio de la moderna sistemática filogenética.

Acrónimos Institucionales: LACM: Los Angeles County Museum of Natural History, Los Angeles, California, Estados Unidos; MCF-PVPH: Museo
'Carmen Funes', Plaza Huincul, Neuquén, Argentina, Paleontología de Vertebrados; MGPIFD-GR: Museo de Geología y Paleontología, Instituto de Formación Docente Continua de General Roca, Río Negro, Argentina.

\section{Geología}

En la región central de la Provincia de Río Negro existe una serie de áreas deprimidas originadas como resultado de la acción combinada de procesos tectónicos y erosivos, en particular deflación (González Díaz y Malagnino, 1984), en donde ocasionalmente existen salinas o salitrales. Precisamente, en el área circundante a uno de ellos, el salitral Ojo de Agua, se han hallado los restos que se describen en este trabajo (Fig. 1). Las sedimentitas cretácicas aflorantes en esta área corresponden a facies terrestres y marino-someras del Grupo Malargüe, depositadas bajo condiciones semiáridas (Barrio, 1990), las cuales se superponen a la Formación Anacleto del Grupo Neuquén (Cretácico Superior) (Hugo y Leanza, 2001). Las facies marinas del Grupo Malargüe están vinculadas a la primera trasgresión atlántica, ocurrida entre el Campaniano medio (Cretácico Tardío alto) y el Daniano (Paleoceno), la cual estableció una profunda bahía, alargada en sentido NNO-SSE (Uliana y Biddle, 1988; Barrio, 1990). La Formación Allen, ubicada en la base del Grupo Malargüe y portadora de los huesos de terópodos que se reportan en esta contribución, está separada del subyacente Grupo Neuquén (CenomanianoCampaniano inferior) por una discordancia erosiva (Andreis et al., 1974). La edad de la Formación Allen, basada en el análisis de ostrácodos (Ballent, 1980), está restringida al Campaniano medioMaastrichtiano temprano. La Formación Allen está compuesta por una gruesa sucesión de areniscas y fangolitas, con intercalaciones de evaporitas (Fig. 2). Salgado et al. (2007b) reconocieron dos subunidades de esta formación, en dos localidades de la Provincia de Río Negro (Salitral de Santa Rosa y Salitral Ojo de Agua): 1. la inferior, que comprende depósitos arenosos de grano fino con capas fangosas subordinadas y niveles evaporíticos delgados y 2 . la superior, una gruesa sucesión de depósitos pelíticos, con intercalaciones de limolitas ricas en ostrácodos, y niveles arenosos.

Sobre la base de sus rasgos sedimentológicos y litofaciales, la unidad inferior, de unos $85 \mathrm{~m}$ de espesor y la que contiene los fósiles de vertebrados, 


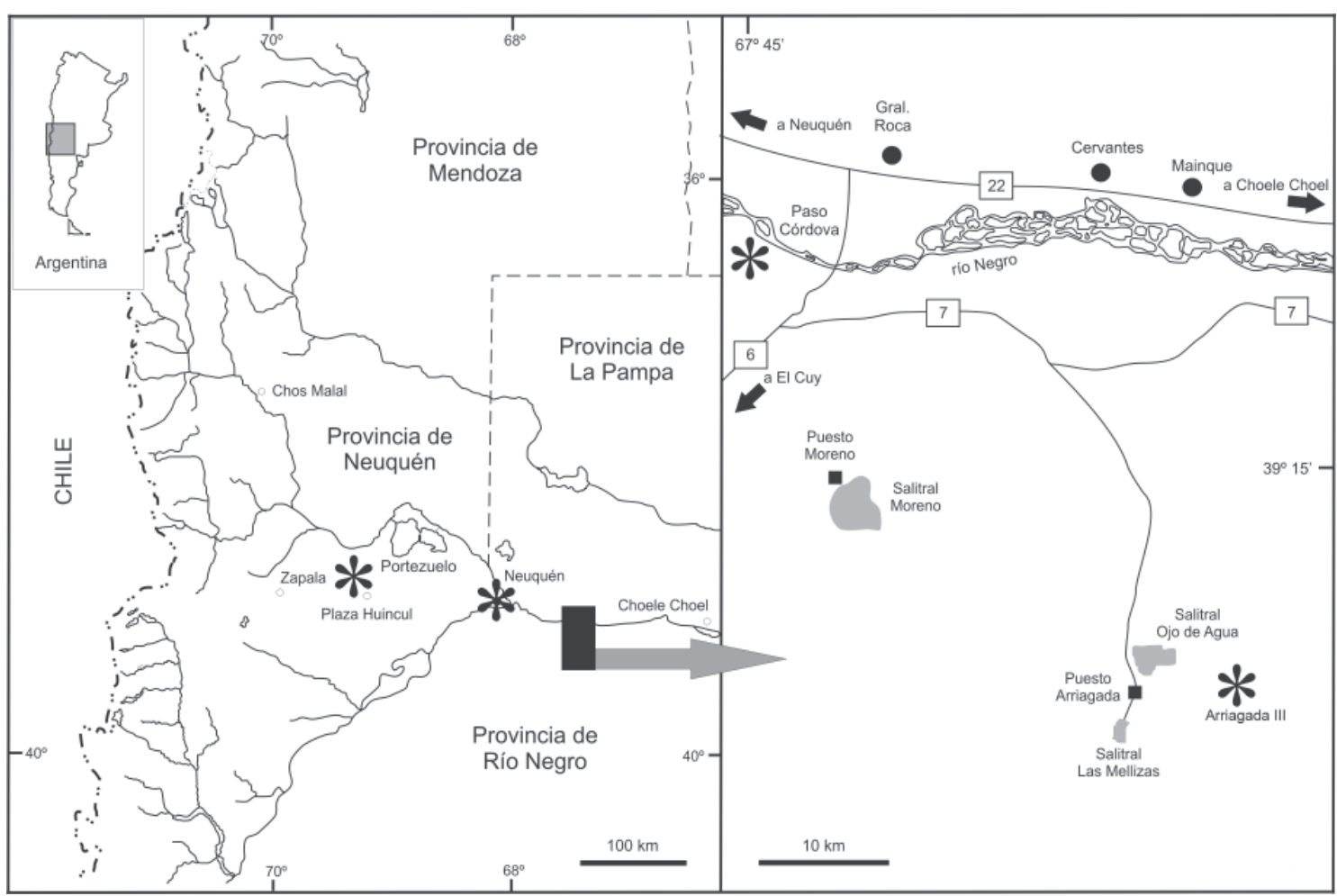

FIG. 1. Mapa de ubicación de la localidad Salitral Ojo de Agua. Las estrellas indican las localidades que han brindado restos de alvarezsáuridos.

fue interpretada por Salgado et al. (2007b) como un lago salobre en un ambiente supramareal, asociado a depósitos de arenas eólicas y de ríos efímeros. El desarrollo de paleosuelos está evidenciado por la abundante rizoturbación, termiteros, así como por la presencia de caliches en los depósitos que contienen los huesos. Los restos de dinosaurios que se describen en este trabajo provienen del yacimiento 'Arriagada III', en la barda sur del salitral Ojo de Agua, donde se expone el 'egg level 2' de Salgado et al. (2007b) (Fig. 1).

\section{Descripciones Sistemáticas}

Theropoda Marsh, 1881

Tetanurae Gauthier, 1986

Coelurosauria Huene, 1920

Maniraptora Gauthier, 1986

Alvarezsauridae Bonaparte, 1991

Gen. et sp. indet.

Material: los materiales en gran parte fragmentados, pertenecientes a un número indeterminado de individuos, fueron recolectados en una superficie de aproximadamente $30 \mathrm{~m}^{2}$; el ejemplar MGPIFDGR 166, correspondiente a un único ejemplar, se encontró asociado a fragmentos de un huevo elongatoolítido. MGPIFD-GR 166 comprende cuatro arcos neurales cervicales posteriores incompletos, una vértebra caudal prácticamente completa (sólo le falta el proceso transverso izquierdo), sector proximal de pubis derecho, fragmentos de costillas e indeterminados; MGPIFD-GR 167, un arco neural cervical (o eventualmente dorsal anterior incompleto; MGPIFD-GR 177, un fragmento de postzigapófisis de una vértebra cervical posterior o una cérvicodorsal; MGPIFD-GR 193, una postzigapófisis cervical; MGPIFD-GR 171, dos cuerpos vertebrales sacrales incompletos fusionados; MGPIFD-GR 170, un cuerpo vertebral caudal anterior; MGPIFD-GR 173, un cuerpo vertebral caudal medio; MGPIFD-GR 168, un fragmento de cuerpo vertebral caudal posterior; MGPIFD-GR 172, un cuerpo vertebral caudal; MGPIFD-GR 175, una porción de arco neural; MGPIFD-GR 176, una prezigapófisis; MGPIFD-GR 179, un fragmento de cuerpo vertebral caudal; MGPIFD- 


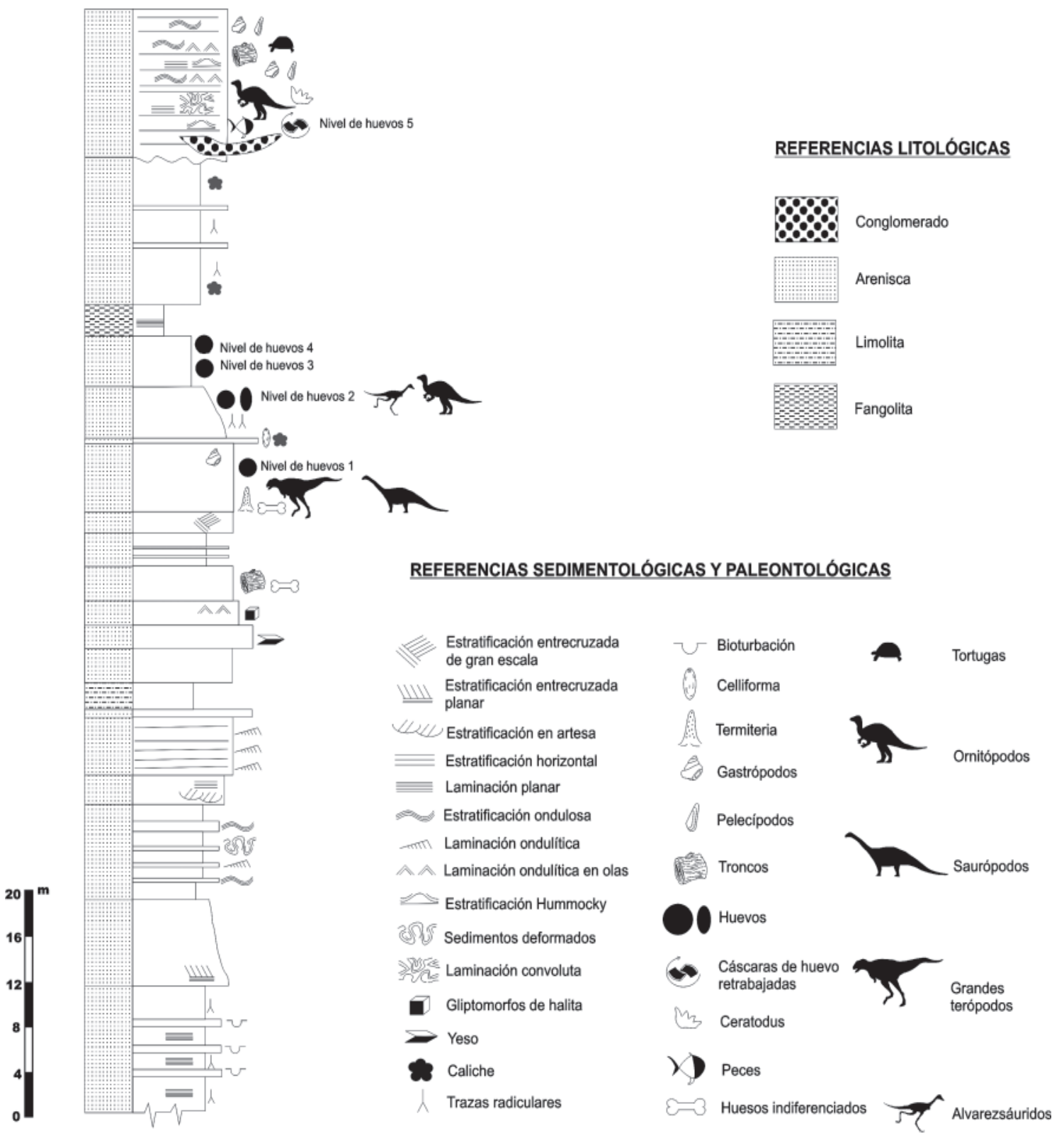

FIG. 2. Perfil estratigráfico integrado de los afloramientos campaniano-maastrichtianos de la Formación Allen en la zona trabajada (modificado de Salgado et al., 2007b).

GR 180 y de un arco neural; MGPIFD-GR 181, fragmentos de un cuerpo vertebral, MGPIFD-GR 183 y MGPIFD-GR 178, un arco hemal; MGPIFD-GR 184, un fragmento de escápula izquierda; MGPIFD-GR 174 y de la articulación proximal de falange pedal; MGPIFD-GR 182, una articulación proximal de falange pedal; MGPIFD-GR 185, una falange pedal completa; MGPIFD-GR 186, un fragmento proximal de una falange pedal; MGPIFD-GR $187,188,189,190$, falanges pedales no ungueales; MGPIFD-GR 191, una falange pedal ungueal casi completa; MGPIFD-GR 192, una falange ungueal pedal incompleta. 
Procedencia geográfica y estratigráfica: Yacimiento 'Arriagada III' (39²5'55”'S; 67¹7'40”O), Salitral Ojo de Agua, Río Negro, Argentina. 'Nivel de huevos 2', Formación Allen (Campaniano medio-Maastrichtiano temprano) (Salgado et al., 2007b) (Figs. 1 y 2).

Descripción: Vértebras cervicales (Fig. 3). Se ha preservado una serie de arcos neurales, no muy alargados, posiblemente correspondientes a vértebras del sector medio a posterior del cuello.

Los canales neurales son muy amplios, de sección transversal subcircular, y levemente deprimidos. En los elementos más anteriores de la sucesión (MGPIFD-GR 166/1), los procesos transversos se proyectan lateralmente, mientras que en la cervical más posterior de la serie recuperada lo hacen dorso-lateralmente (MGPIFD-GR 167). La espina neural es relativamente corta anteroposteriormente y orientada verticalmente.

En ningún caso se han conservado las parapófisis, ya que, como ocurre en los cuellos de la mayoría de los terópodos, las mismas se ubican en los sectores laterales de los cuerpos vertebrales. De esta manera, la orientación vertical de la lámina centrodiapofisial anterior (acdl) que se observa en MGPIFD-GR 166/1 y MGPIFD-GR 166/2, revela que la parapófisis habría ocupado una posición más ventral que en MGPIFD-GR 167 (Fig. 3G, H), en donde esa lámina es paralela a la prezigodiapofisial (prdl), lo que sugiere una posición más posterior en la secuencia. En Alvarezsaurus, aun en la vértebra dorsal 3, las parapófisis se disponen en una posición muy baja (Bonaparte, 1991, fig. 23), mientras que en los alvarezsáuridos asiáticos, por el contrario, dichas estructuras se presentan, apomórficamente, a la altura de las prezigapófisis (Chiappe et al., 2002). Por lo tanto, MGPIFD-GR 167 podría corresponder a una vértebra cervical muy posterior, posiblemente una cérvicodorsal, o incluso una dorsal anterior.

Sobre la cara posterior de los procesos transversos se desarrolla una fosa infrapostzigapofisial, como en los alvarezsáuridos asiáticos (Chiappe et al., 2002, fig. 4.11); la misma parece ser menos profunda en la cervical interpretada como

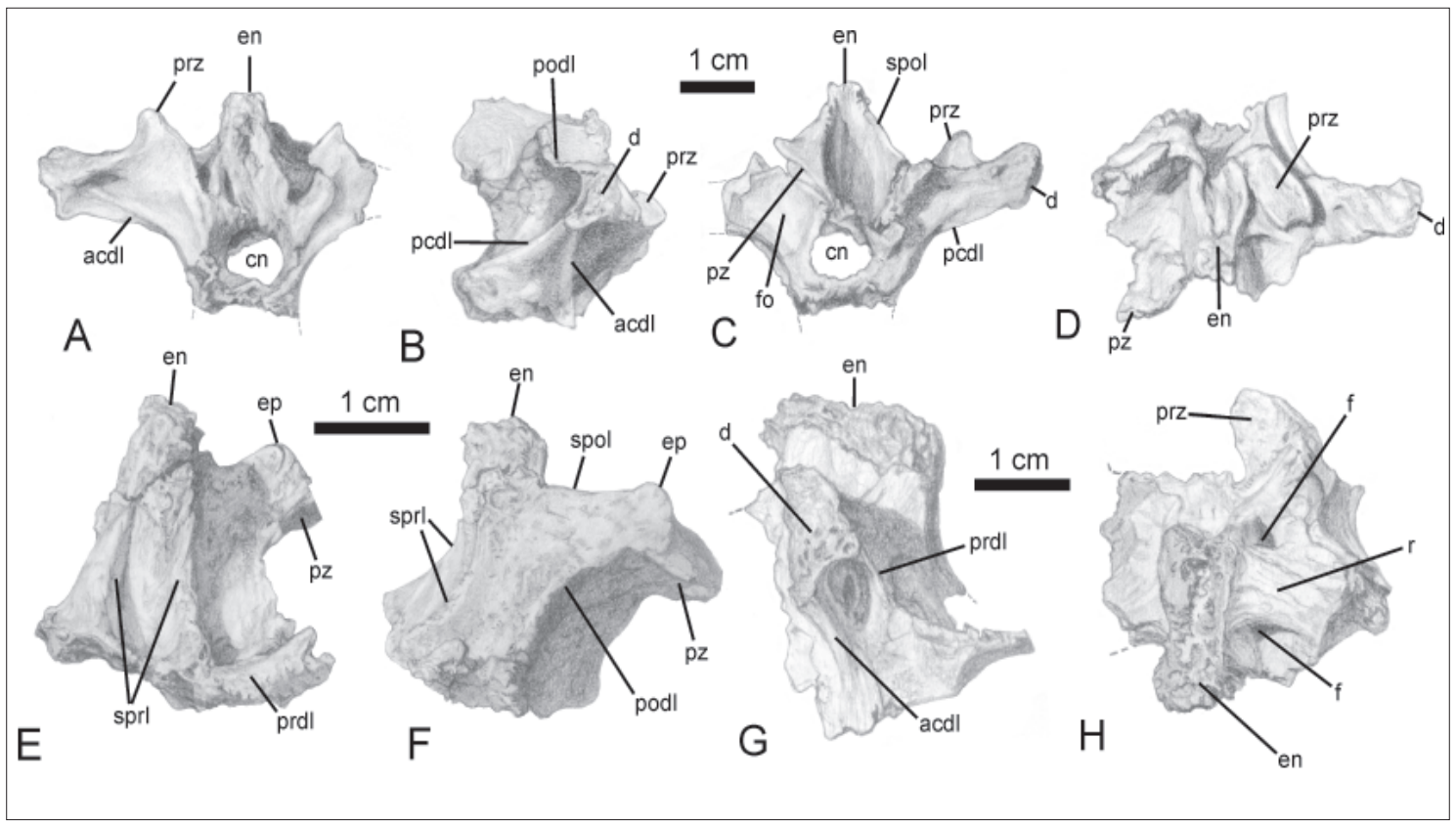

FIG. 3. Vértebras cervicales del Alvarezsauridae indet. en 'Arriagada III', Salitral Ojo de Agua. MGPIFD-GR 166/1 en vista anterior (A), lateral derecha (B), posterior (C), y anterodorsal (D). MGPIFD-GR 166/3 en vista anterior (E) y lateral izquierda (F). MGPIFD-GR 167 en vista lateral derecha (G) y dorsal (H). Abreviaturas: acdl. lámina centrodiapofisial anterior; cn. canal neural; d. diapófisis; en. espina neural; ep. epipófisis; f. foseta; fo. fosa infrapostzigapofisial; pedl. lámina centrodiapofisial posterior; podl. lámina postzigodiapofisial; prdl. lámina prezigodiapofisial; prz. prezigapófisis; pz. postzigapófisis; r. reborde; spol. lámina espinopostzigapofisial; sprl. lámina espinoprezigapofisial. 
más posterior (MGPIFD-GR 167). Las superficies de articulación de las prezigapófisis son planas y expandidas transversalmente en las vértebras más anteriores (MGPIFD-GR 166/1), mientras que en las más posteriores, las mismas parecen estar expandidas anteroposteriormente (MGPIFD-GR 167). Las postzigapófisis están expandidas en forma transversal, y los planos de las carillas articulares convergen ventromedialmente. En vista dorsal, los extremos distales de las postzigapófisis se proyectan de manera lateral (MGPIFD-GR 166/1). En las postzigapófisis de MGPIFD-GR 166/1/2/3, sobre el borde anterolateral de las mismas, se observan las epipófisis bien desarrolladas (Fig. 3E, F), similares a las del ejemplar MCF-PVPH 38, el cual estaba asociado al holotipo de Patagonykus puertai, pero considerado un alvarezsáurido indeterminado por Novas (1997), y a las de Mononykus y Alvarezsaurus. No obstante, las epipófisis de los alvarezsáuridos de la Formación Allen, así como las del ejemplar MCF-PVPH 38, están más desarrolladas que en esos dos últimos géneros.

Los arcos neurales recuperados carecen del complejo articular hiposfenohipantro. Estas estructuras están ausentes también en las formas asiáticas (Chiappe et al., 2002), contrariamente a lo mencionado para Patagonykus (Novas, 1997), aunque su existencia en este último caso es dudosa.

En los materiales de la Formación Allen, el sistema de láminas óseas que conectan los distintos puntos del arco neural se encuentra bien desarrollado, más que en Alvarezsaurus, en donde la lámina paradiapofisial $(\mathrm{ppdl})$ recién comienza a notarse a partir de la tercera dorsal (Bonaparte, 1991, fig. 23). La lámina centrodiapofisial posterior (pcdl) se dirige de manera posteroventral, uniéndose distalmente a la lámina centropostzigapofisial (cpol) en el ángulo posteroventral del arco neural. La lámina postzigodiapofisial (podl) está muy bien desarrollada en las cervicales. Al menos en las más anteriores, esta última lámina parece ser más bien horizontal y tiene un desarrollo mayor que la lámina prezigodiapofisial (prdl). En las cervicales posteriores (MGPIFD-GR 166/3, MGPIFD-GR 167), como resultado de la proyección laterodorsal del proceso transverso $\mathrm{y}$ el consecuente ascenso de la diapófisis, la podl se orienta oblicuamente desde el proceso transverso en sentido posteroventral (Fig. 3A-H).

La espina neural de las vértebras cervicales está formada por las láminas espinoprezigapofisiales (sprl) y espinopostzigapofisiales (spol). En la cervical más anterior se observa una lámina que ocupa una posición similar a la espinodiapofisial (spdl) de los neosaurópodos (Wilson, 1999), la cual se encuentra flanqueada anterior y posteriormente por sendas fosetas laterales a la base de la espina neural. En la cervical más posterior, esta lámina se presenta como un grueso reborde que ocupa prácticamente toda la superficie dorsal del proceso transverso, e igualmente situado entre dos fosetas (Fig. 3H).

Vértebras sacrales (Fig. 4). Los dos cuerpos vertebrales sacrales preservados están incompletos y fuertemente fusionados entre sí (MGPIFD-GR 171). A diferencia de las sacrales posteriores lateralmente comprimidas de otros Alvarezsauridae (Chiappe et al., 2002), el material de la Formación Allen presenta una sección subcilíndrica, más comparable a Alvarezsaurus que a Patagonykus y Mononykus. Tampoco presenta una quilla ventral en las sacrales caudales, como sí ocurre en Patagonykus (Novas, 1997) y Mononykus (Chiappe et al., 2002). En este sentido, MGPIFD-GR 171 es nuevamente semejante a las primeras sacrales de Alvarezsaurus (Bonaparte, 1991). En el alvarezsáurido de la Formación Allen, sobre la cara ventral de ambos cuerpos sacrales, se observa una ligera depresión longitudinal. Teniendo en cuenta la presencia de este carácter en Alvarezsaurus, MGPIFD-GR 171 correspondería a los cuerpos sacrales 2 y 3 .

Vértebras caudales (Fig. 5). Se han recuperado seis fragmentos de cuerpos caudales correspondientes a diferentes sectores de la cola y una vértebra caudal anterior o media prácticamente completa (MGPIFD-GR 166/4, Fig. 5C, D). Los demás fragmentos, los mejor preservados, son, en orden secuencial: MGPIFD-GR 170 (Fig. 5A, B) y 172 (caudales anteriores), MGPIFD-GR 173 (caudal media), MGPIFD-GR 168 (caudal medio-distal). Todos los cuerpos vertebrales son procélicos, como en Mononykus, Alvarezsaurus, Patagonykus y el recientemente descrito Achillesaurus manazzonei Martinelli y Vera, 2007, y muy comprimidos lateralmente, sobre todo en MGPIFD-GR 166/4 (Fig. $5 \mathrm{C}, \mathrm{D})$. Las caras laterales de los cuerpos vertebrales son relativamente altas, y levemente cóncavas anteroposteriormente, sin que se observe la pequeña depresión subcircular presente en Achillesaurus. En todos los cuerpos caudales recuperados, el arco neural es anteroposteriormente extendido y ocupa toda la longitud del cuerpo vertebral. Los cuerpos vertebrales caudales más anteriores son prácticamente tan altos como largos, mientras que en Patagonykus la longitud es el doble de la altura (Novas, 1997). 


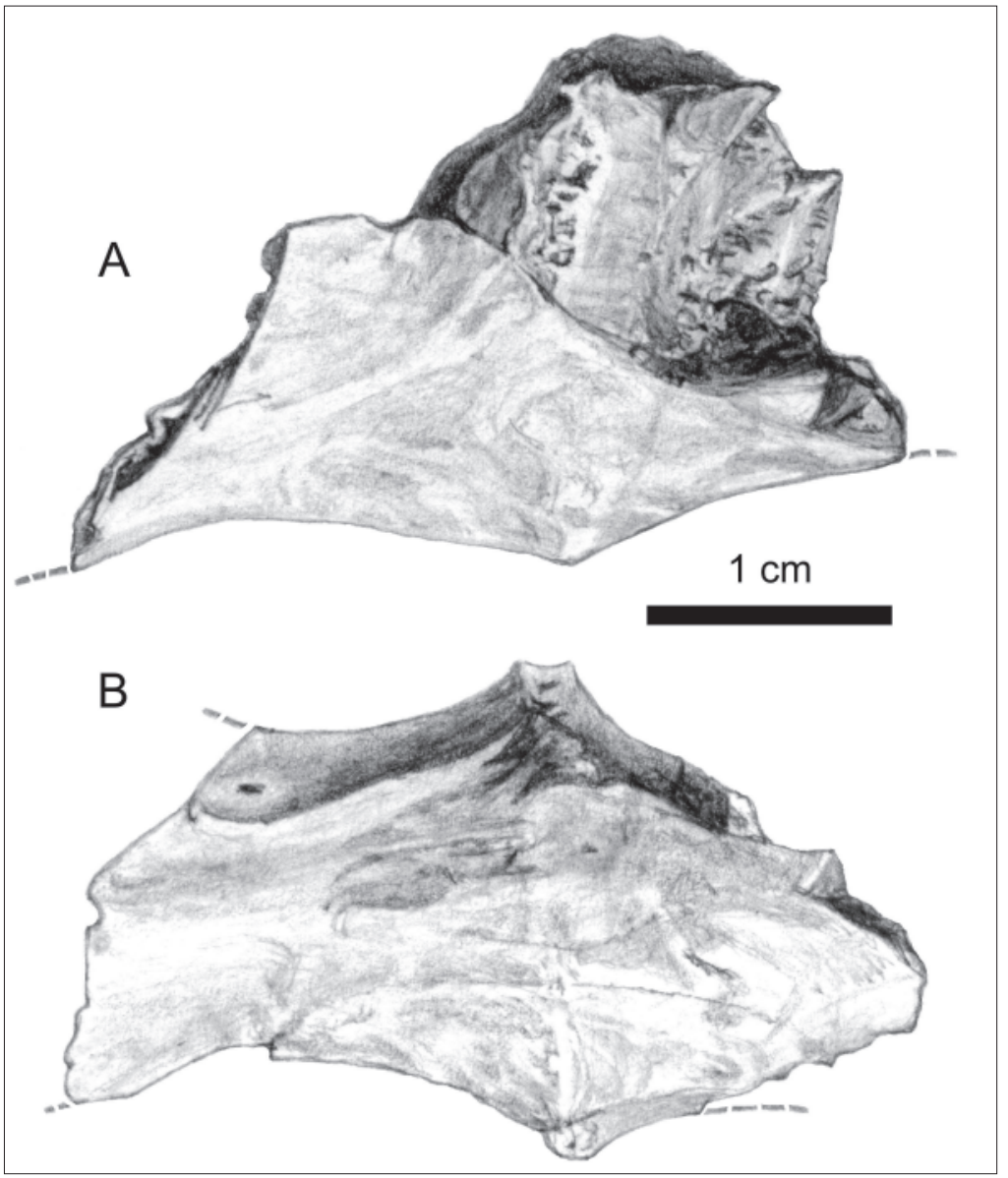

FIG. 4. Vértebras sacrales del Alvarezsauridae indet. en 'Arriagada III', Salitral Ojo de Agua. MGPIFD-GR 171 en vistas lateral (A) y ventral (B).

En los ejemplares MGPIFD-GR 172 y 173 que han conservado la parte posterior de la cara ventral del cuerpo caudal, ésta es muy angosta y flanqueada por dos rebordes longitudinales. En la parte media del cuerpo vertebral, esos rebordes se aproximan mucho hasta casi contactar, dejando un surco entre ellos, como se observa en Shuvuuia (Chiappe et al., 2002) y en un ejemplar inédito de la Formación Hell Creek de los Estados Unidos (LACM 153311). La cara ventral del cuerpo caudal MGPIFD-GR 166/4 presenta un afilado reborde longitudinal, horizontal en vista lateral a modo de quilla (Fig. 5D).

En todos los cuerpos caudales colectados, la articulación anterior (cóncava) es más amplia que la articulación posterior (convexa), tal vez por la presencia de un anillo cartilaginoso perimetral a la articulación posterior. En ningún caso se observa el promontorio o bulbo lateroposterior identificado en Patagonykus (Novas, 1997).
La vértebra caudal completa (MGPIFD-GR166/4) podría corresponder a la quinta o sexta de la serie, sobre la base de lo conocido para Shuvuuia (Chiappe et al., 2002, fig. 4.13). Las prezigapófisis son amplias, con las carillas articulares de contorno oval, con el eje mayor del óvalo dirigido anterolateralmente, y alojadas en planos que convergen ventralmente. La espina neural es baja, mientras que las postzigapófisis son pequeñas y proyectadas lateralmente. Los procesos transversos son planos y se sitúan a nivel de la mitad caudal del cuerpo (sin contar la articulación caudal) como en Alvarez-saurus calvoi Bonaparte, 1991. Ventralmente al proceso transverso, se observa un reborde longitudinal que recorre el cuerpo vertebral en sentido anteroposterior. El cuerpo vertebral está fuertemente comprimido en su cara lateralmente, presentando un afilado borde ventral portador de un surco longitudinal. La cara articular anterior del cuerpo es fuertemente 


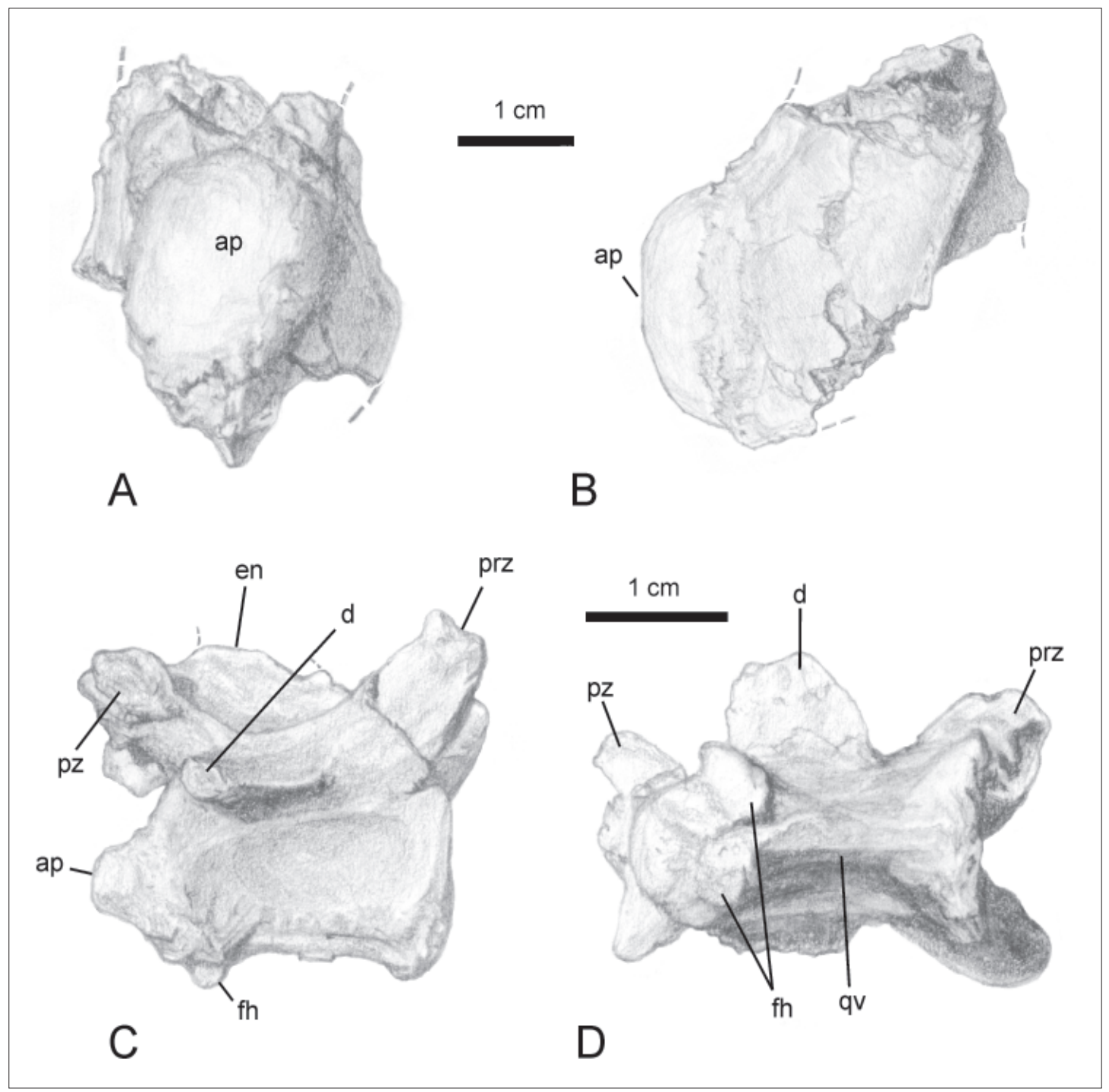

FIG. 5. Vértebras caudales del Alvarezsauridae indet. en 'Arriagada III', Salitral Ojo de Agua. MGPIFD-GR 170, vértebra caudal anterior en vista posterior (A) y lateral derecha (B); MGPIFD-GR 166/4, vértebra caudal anterior o media en vista lateral derecha (C) y ventral (D). Abreviaturas: ap. articulación posterior; d. diapófisis; en. espina neural; prz. prezigapófisis; pz. postzigapófisis; fh. faceta para las hemapófisis; qv. quilla ventral.

cóncava, de contorno oval, más alta que ancha, y el cóndilo articular posterior es asimétrico en vista lateral y se encuentra ubicado dorsalmente sobre la cara articular.

Escápula (Fig. 6A). Se ha recuperado un fragmento de escápula izquierda (MGPIFD-GR 184) con rasgos similares a los de la escápula de Mononykus olecranus Perle, Norell, Chiappe y Clark, 1993 (Perle et al., 1994). El mismo es comprimido la- teralmente, curvado medialmente, con el extremo proximal expandido dorsoventralmente y con los bordes dorsal y ventral subparalelos en vista lateral. La cara lateral es convexa, mientras que la cara medial se encuentra recorrida longitudinalmente por un surco somero, que la divide en un sector dorsal y otro ventral, cuyas superficies son convexas en sentido dorsoventral, lo que determina una sección transversal de contorno arriñonado. El borde latero- 


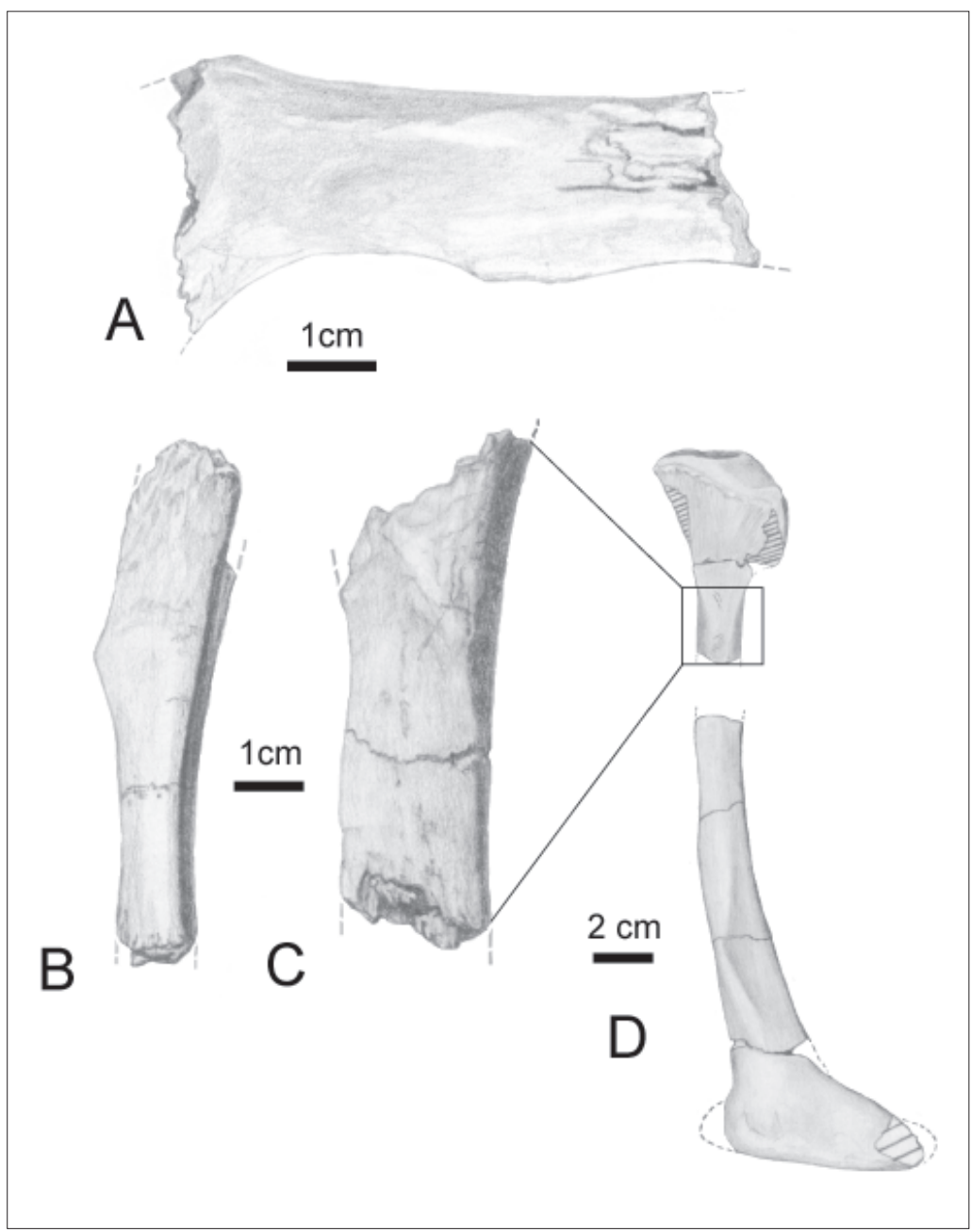

FIG. 6. (A) escápula izquierda del Alvarezsauridae indet. en 'Arriagada III', Salitral Ojo deAgua. MGPIFD-GR 184 en vista lateral; (B) pubis izquierdo del Alvarezsauridae indet. de Salitral Ojo de Agua (MGPIFD-GR 194) en vista anterior (B) y medial (C); (D) Patagonykus puertai, holotipo MCF-PVPH 37, pubis derecho en vista medial.

ventral del extremo proximal está engrosado transversalmente. Sobre el borde ventral se observa un pequeño proceso afilado, suavemente convexo, que también está presente en la escápula de Mononykus (Perle et al., 1994, fig. 9).

Pubis (Fig. 6B, C). El espécimen MGPIFD-GR 194 es un fragmento proximal de un pubis izquierdo, que representaría un animal de mayor tamaño que el holotipo de Patagonykus puertai (Novas, 1997). La sección transversal de la pieza es oval, como en Patagonykus y Mononykus, mientras que en Shuvuuia es subcircular (Suzuki et al., 2002). Su superficie medial es lisa, ligeramente cóncava, en tanto que la lateral es suavemente convexa. Sobre el borde anteromedial se observa un tubérculo o promontorio, vinculado a un reborde óseo que recorre la cara medial en sentido oblicuo (Fig. 6C).
Esta prominencia es más desarrollada que la de Patagonykus (Fig. 6D), probablemente debido a la significativa diferencia de tamaño. La misma posiblemente corresponda al tubérculo para la inserción del músculo ambiens (Hutchinson, 2001).

Falanges pedales (Fig. 7). Se han recuperado cinco falanges pedales no ungueales, MGPIFD-GR 185, III.1 izquierda; MGPIFD-GR 187, II.1 izquierda; MGPIFD-GR 188, IV.2? derecha; MGPIFD-GR 190, IV.3? derecha; MGPIFD-GR 189, IV.4? derecha. Las tres últimas falanges están incompletas. También se recolectaron varios extremos proximales de falanges indeterminables (MGPIFD-GR 174 y MGPIFD-GR 186) y tres falanges ungueales (MGPIFD-GR 191, 192 y 194).

La falange MGPIFD-GR 185 (Fig. 7A-D) es una pieza alargada con profundas y diminutas fo- 


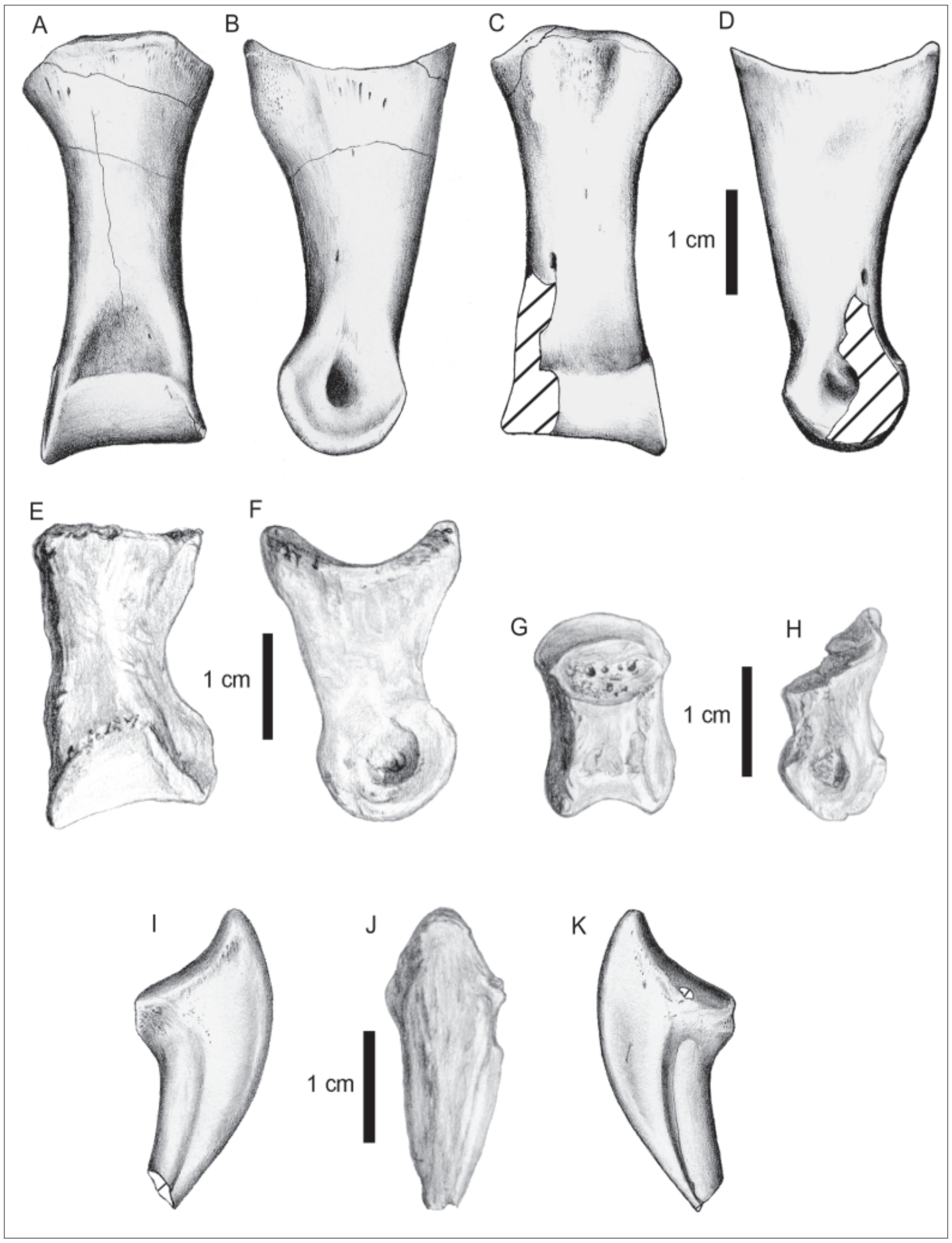

FIG. 7. Falanges del Alvarezsauridae indet. en 'Arriagada III', Salitral Ojo de Agua. MGPIFD-GR 185, falange pedal III.1, izquierda en vista dorsal (A), medial (B), ventral (C) y lateral (D). MGPIFD-GR 187, falange pedal II.1 izquierda en vista dorsal (E) y medial (F). MGPIFD-GR 190, falange pedal IV.3? derecha en vista dorsal (G) y medial (H). MGPIFD-GR 191 falange ungueal derecha? en vista lateral (I), dorsal (J) y medial (K). 
setas ligamentarias a ambos lados de la articulación ginglimoide. La articulación proximal tiene un contorno ligeramente pentagonal. La fosa extensora está bien marcada y presenta un contorno triangular, de vértice proximal, cuya base la constituye el margen proximal del extremo articular. En vista distal, el extremo articular parece ser casi simétrico, expandido en sus extremos y comprimido en su parte media.

La falange MGPIFD-GR 187, interpretada como la falange II.1 izquierda (Fig. 7E, F), posee la foseta lateral más profunda del lado medial, así como el perímetro del sector articular más extenso que el del lado opuesto, como en Patagonykus (Novas, 1997). En vista dorsal, se observa una marcada torsión medial de la pieza, como la observada en Mononykus (Perle et al., 1994). La superficie articular proximal es cóncava, mientras que la fosa extensora está menos desarrollada que en la falange MGPIFD-GR 185.

La falange MGPIFD-GR 188, posiblemente una falange IV.2, es relativamente corta y su articulación proximal es apenas más ancha que alta, convexa en sentido del eje transversal y marcadamente cóncava dorsoventralmente. MGPIFD-GR 190, posiblemente una falange IV.3 derecha, es una pieza apenas más larga que ancha, con extremos articulares proporcionalmente grandes y una fosa extensora relativamente profunda (Fig. 7G, H).

Se han recuperado tres falanges pedales ungueales, una de las cuales se encuentra prácticamente completa (MGPIFD-GR 191, Fig. 7I-K). En esta última, la cara lateral es más convexa que la medial, mientras que la cara ventral y la articular son planas. En vista dorsal, se observa que la pieza está curvada medialmente, con el borde dorsal proyectado laterodorsalmente en vista anterior (Fig. 7J), lo que le confiere un aspecto asimétrico. Sobre las caras laterales se observan sendos surcos o acanaladuras que pierden profundidad hacia el extremo proximal o posterior. De ambos, el surco externo es el más profundo; éste parece estar bifurcado proximalmente como ocurre en algunos alvarezsáuridos (ej. Patagonykus), y en algunos abelisaurios (Novas y Bandyopadhyay, 2001). El lateral 'bump' determinado por ambas bifurcaciones del surco es casi plano, como en los demás coelurosaurios. Otra falange ungueal (MGPIFD-GR 174), más fragmentaria y meteorizada, muestra un surco simple sobre la cara lateral y una cara medial lisa. Si bien está incompleta, es sin duda una falange pedal, porque no es muy comprimida lateralmente como las manuales.

\section{Discusión}

Los materiales recolectados en Salitral Ojo de Agua presentan al menos uno de los caracteres diagnósticos propuestos por Novas (1997) para los Alvarezsauridae: cuerpos caudales procélicos. Los restos descritos corresponden posiblemente a una nueva especie de alvarezsáurido, ya que todos proceden de un área relativamente reducida (unos $30 \mathrm{~m}^{2}$ ), son morfológicamente homogéneos y constituyen el primer registro de Alvarezsauridae para la Formación Allen. Otros restos aún sin describir, y que provendrían de la misma área (ver Agnolin et al., 2006), podrían corresponder a la misma especie, aunque aún debe esperarse un estudio definitivo de esos materiales.

Los materiales aquí descritos de Salitral Ojo de Agua son fragmentarios, y carecen de las autapomorfías propuestas para Patagonykus. De todas formas, en términos generales, los alvarezsáuridos de la Formación Allen son morfológicamente más similares a este género que a otros alvarezsáuridos. Es obviamente necesario el hallazgo de más material que permita realizar una estimación más precisa de las relaciones de este taxón.

Además de la condición procélica de sus cuerpos caudales, común a la mayoría de los alvarezsáuridos, los materiales de Salitral Ojo de Agua presentan, como Shuvuuia, los cuerpos caudales muy comprimidos transversalmente y portadores de un surco longitudinal en su cara ventral. A su vez, comparte con Patagonykus la presencia de epipófisis cervicales que no superan el margen posterior de las postzigapófisis.

Los fósiles de Salitral Ojo de Agua aquí descritos provienen de la Formación Allen, a la cual se le atribuye una edad campaniano medio-maastrichtiano temprano. De esta forma, los alvarezsáuridos descritos en este trabajo serían aproximadamente contemporáneos, o ligeramente más antiguos que las formas asiáticas de la Formación Nemegt (Cretácico Tardío alto): Mononykus y Shuvuuia (Chiappe et al., 2002).

Todos los análisis realizados hasta la fecha han concluido que Patagonykus de la localidad de Portezuelo (Provincia de Neuquén) y de la Formación Portezuelo (de edad turoniano tardíoconiaciana temprana, Hugo y Leanza, 2001) estaría más cercanamente relacionado con el género asiático Mononykus que con Alvarezsaurus (registrado en la ciudad de Neuquén, a unos $90 \mathrm{~km}$ al NO de Salitral Ojo de Agua) y Achillesaurus 
(registrado en Paso Córdova, Provincia de Río Negro, a unos $45 \mathrm{~km}$ al NO de Salitral Ojo de Agua) (Novas, 1997; Chiappe et al., 2002; Martinelli y Vera, 2007). Estos últimos proceden de la misma cuenca sedimentaria que Patagonykus, pero de niveles más modernos, pertenecientes a la Formación Bajo de la Carpa (Santoniano, Leanza et al., 2004). Esta hipótesis, que vincula a Mononykus con Patagonykus en un grupo monofilético y excluye al resto de los alvarezsáuridos, impide proponer la existencia de una radiación de este clado con integrantes estrictamente sudamericanos.

Los restos de los terópodos que se describen aquí se hallaban asociados a cuatro tipos diferentes de cáscara de huevos, el más abundante de los cuales es uno asignado a la oofamilia Elongatoolithidae (Zhao, 1975), atribuido generalmente a los Theropoda (Salgado et al., 2007b). En un caso (MGPIFDGR 166), los restos óseos estaban entremezclados con cáscaras de huevo de ese tipo. Sin embargo, la presencia conjunta de restos óseos de alvarezsáuridos y abundantes cáscaras de elongatoolítidos no es evidencia suficiente para identificar a estos terópodos como productores de dichos huevos; sólo se podrá arribar a una conclusión con el hallazgo de material embrionario.

El conocimiento sobre la diversidad de terópodos en Patagonia durante los últimos 10 millones de años del Cretácico se ha incrementado significativamente en años recientes (Coria, 2007). A Carnotaurus sastrei Bonaparte, 1985, hasta hace unos años el único terópodo conocido de este período, se han agregado recientemente el probable Tetanurae Quilmesaurus curriei Coria, 2001 (ver Juárez Valieri et al., 2007, para una propuesta taxonómica alternativa), un diente fragmentario asignado tentativamente a Carcharodontosauridae (Martinelli y Forasiepi, 2004), un probable Dromaeosauridae (Novas et al., 2003) y restos de otros tetanuros indeterminados (Coria y Salgado, 2005). Si se consideran los restos finicretácicos del NO argentino, deben incluirse el abelisauroideo Noasaurus leali Bonaparte y Powell, 1980, el Maniraptora Unquillosaurus ceibali Powell, 1979 (Novas y Agnolin, 2004) y un posible Oviraptorosauria (Frankfurt y Chiappe, 1999; véase Agnolin y Martinelli, 2007, para una alternativa taxonómica).

Los alvarezsáuridos de Salitral Ojo de Agua descritos en este trabajo amplían el registro de esta familia al Cretácico Tardío alto de la Argentina; además, extienden al Cretácico continental más tardío de Patagonia el biocrón de este particular grupo de terópodos celurosaurios.

\section{Agradecimientos}

Los trabajos han sido financiados por la 'National Geographic Society' (grant 7396/03), el Conicet (PIP 6455) y la Agencia Nacional de Promoción Científica y Técnica (PICT-2006-00357). Los autores desean agradecer a K. Curry Rogers ('Science Museum of Minnesota', Saint Paul, Minnesota, EE.UU.), R. Rogers (Macalester College, Saint Paul, Minnesota, EE.UU.), R. Ortiz (Museo de Geología y Paleontología del Instituto de Formación Docente Continua de General Roca, Río Negro, Argentina), M. Talevi (Universidad Nacional de Rosario, Santa Fe, Argentina), I. Cares (colaborador por el Museo Provincial de Cipolletti, Río Negro, Argentina), y A. Garrido (Museo 'Profesor Dr. Juan Olsacher', Zapala, Neuquén, Argentina) por su participación en la campaña. Asimismo, los autores agradecen a la familia Arriagada por su hospitalidad durante nuestra estadía en Salitral Ojo de Agua. A P. Pacheco por los dibujos.

\section{Referencias}

Agnolin, F.L.; Martinelli, A.G. 2007. Did oviraptorosaurs (Dinosauria; Theropoda) inhabit Argentina? Cretaceous Research 28: 785-790.

Agnolin, F.; Powell, J.E.; Novas, F.E. 2006. Discovery of Maastrichtian alvarezsaurid theropods in NW Patagonia, Argentina. In Jornadas Argentinas de Paleontología de Vertebrados, No. 22, Resúmenes: p. 6. San Juan.

Albino, A.M. 1986. Nuevos Boidae Madtsoiinae en el Cretácico Tardío de Patagonia (Formación Los Alamitos, Río Negro, Argentina). In Congreso Argentino de Paleontología y Bioestratigrafía, No. 4, Actas 2: 15-21. Mendoza.

Andreis, R.A.; Iñíguez,A.M.; Rodríguez Lluch, J.J.; Sabio, D.A. 1974. Estudio sedimentológico de las formaciones del Cretácico Superior del área del Lago Pellegrini (Prov. de Río Negro, Rep. Argentina). Revista de la Asociación Geológica Argentina 29: 85-104.

Ballent, S.C. 1980. Ostrácodos de ambiente salobre de la Formación Allen (Cretácico Superior) en la Provincia de Río Negro (República Argentina). Ameghiniana 17 (1): 67-82.

Barrio, C.A. 1990. Paleogeographic control of Upper Cretaceous tidal deposits, Neuquén Basin, Argentina. Journal of South America Earth Sciences 3: 31-49.

Bonaparte, J.F. 1985. A horned Cretaceous carnosaur from Patagonia. National Geographic Research 1: 150-152.

Bonaparte, J.F. 1987. The Late Cretaceous fauna of Los Alamitos, Patagonia, Argentina. Revista Museo Argentino de Ciencias Naturales 3: 103-179.

Bonaparte, J.F. 1991. Los vertebrados fósiles de la Formación Río Colorado, de la ciudad de Neuquén y 
cercanías, Cretácico Superior, Argentina. Revista del Museo Argentino de Ciencias Naturales 'Bernardino Rivadavia' 4: 16-123.

Bonaparte, J.F.; Powell, J.E. 1980. A continental assemblage of tetrapods from the Upper Cretaceous beds of El Brete, northwestern Argentina (Sauropoda, Coelurosauria, Carnosauria, Aves). Mémoires de la Société Geólogique de France 139: 19-28.

Bonaparte, J.F.; Franchi, M.R.; Powell J.E.; Sepúlveda, E.C. 1984. La Formación Los Alamitos (Campaniano-Maastrichtiano) del sudoeste de Río Negro, con descripción de Kritosaurus australis nov. sp. (Hadrosauridae). Significación paleobiogeográfica de los vertebrados. Revista de la Asociación Geológica Argentina 39: 284-299.

Casamiquela, R.M. 1964. Sobre un dinosaurio hadrosáurido de la Argentina. Ameghiniana 3: 285-312.

Chiappe, L.M.; Norell, M.A.; Clark, J.M. 2002. The Cretaceous, short-armedAlvarezsauridae Mononykus and its kin. In Mesozoic Birds: Above the Head of Dinosaurs (Chiappe, L.M.; Witmer, L.M.; editors). University of California Press: 87-120. Berkeley.

Clark, J.M.; Norell, M.A.; Makovicky, P.J. 2002. Cladistic approaches to the relationships of birds to other theropod dinosaurs. In Mesozoic Birds: Above the Heads of Dinosaurs (Chiappe, L.M.; Witmer, L.M.; editors). University of California Press: 31-60. Berkeley.

Coria, R.A. 2001. A new theropod from the Late Cretaceous of Patagonia. In Mesozoic Vertebrate Life (Tanke, D.H.; Carpenter, K.; editors). Indiana University Press: 3-9. Bloomington.

Coria, R.A. 2007. Nonavian Theropods. In Patagonian Mesozoic Reptiles (Gasparini, Z.; Salgado, L.; Coria, R.A.; editors). Indiana University Press: 229-256. Bloomington.

Coria, R.A.; Salgado, L. 2005. Last Patagonian non-avian theropods. In The Carnivorous Dinosaurs (Carpenter, K.; editor). Indiana University Press: 153-160. Bloomington.

Coria, R.A.; Cambiaso, A.V.; Salgado, L. 2007. New records of basal ornithopod dinosaurs in the Cretaceous of North Patagonia. Ameghiniana 44: 473-477.

De la Fuente, M.S.; de Lapparent de Broin, F.; Manera de Bianco, T. 2001. The oldest and first nearly complete skeleton of a chelid, of the Hydromedusa sub-group (Chelidae, Pleurodira), from the Upper Cretaceous of Patagonia. Bullettin de la Société Géologique de France 172: 237-244.

Frankfurt, N.G.; Chiappe, L.M. 1999. A possible oviraptorosaur from the Late Cretaceous of Northwestern Argentina. Journal of Vertebrate Paleontology 19: 101-105.

Gauthier, J.A. 1986. Saurischian monophyly and the origin of birds. In The Origin of Birds and the Evolution of Flight (Padian, K.; editor). Memoirs of the California Academy of Sciences 8: 1-55.

Göhlich, U.B.; Chiappe, L.M. 2006. A new carnivorous dinosaur from the Late Jurassic Solnhofen archipielago. Nature 440: 329-332.

González Díaz, E.F.; Malagnino, E.C. 1984. Geomorfología de la Provincia de Río Negro. In Congreso Geológico Argentino, No. 9, Publicación Especial, Actas: 1-159. San Carlos de Bariloche.

Huene, F. 1920. Stammesgeschichtliche Ergebnisse einiger Untersuchungen an Trias-Reptilien. Zeitschrift für Induktive Abstammungsund Vererbungslehre 24: 159-163.

Hugo, C.A.; Leanza, H.A. 2001. Hoja Geológica 3966III, Villa Regina, Provincia de Río Negro. Instituto de Geología y Recursos Naturales. Servicio Geológico y Minero de Argentina (SEGEMAR), Boletín 309: $1-53$.

Hutchinson, J.R. 2001. The evolution of pelvic osteology and soft tissues on the line to extant birds (Neornithes). Zoological Journal of the Linnean Society 131: 123-168.

Hwang, S.H.; Norell, M.A.; Gao, K. 2002. New specimens of Microraptor zhaoianus (Theropoda: Dromaeosauridae) from northeastern China. American Museum Novitates 3381: 1-44.

Juárez Valieri, R.; Fiorelli, L.E.; Cruz, L.E. 2007. Quilmesaurus curriei Coria, 2001 (Dinosauria, Theropoda). $\mathrm{Su}$ validez taxonómica y relaciones filogenéticas. Revista del Museo Argentino de Ciencias Naturales 9: 59-66.

Leanza, H.A.; Apesteguía, S.; Novas, F.E.; de la Fuente, M.S. 2004. Cretaceous terrestrial beds from Neuquén Basin (Argentina) and their tetrapod assemblages. Cretaceous Research 25: 61-87.

Makovicky, P.J.; Apesteguía, S.; Agnolin, F.L. 2005. The earliest dromaeosaurid theropod from South America. Nature 437: 1007-1011.

Marsh, C.O. 1881. Principal characters of American Jurassic dinosaurs. American Journal of Science 21 (3): 417-423.

Martinelli, A.G.; Forasiepi, A.M. 2004. Late Cretaceous vertebrates from Bajo de Santa Rosa (Allen Formation), Río Negro province, Argentina, with the description of a new sauropod dinosaur (Titanosauridae). Revista del Museo Argentino de Ciencias Naturales 6: 257-305.

Martinelli, A.G.; Vera, E.I. 2007. Achillesaurus manazzonei, a new alvarezsaurid theropod (Dinosauria) from the Late Cretaceous Bajo de la Carpa Formation, Río Negro Province, Argentina. Zootaxa 1582: 1-17.

Norell, M.A.; Clark, J.M.; Makovicky, P.J. 2001. Phylogenetic relationships among coelurosaurian dinosaurs. In New perspectives on the Origin and Evolution of Birds (Gauthier, J.; Gall, L.F.; editors). Yale University Press: 49-67. New Haven.

Novas, F.E. 1997. Anatomy of Patagonykus puertai (Theropoda, Avialae, Alvarezsauridae), from the Late Cretaceous of Patagonia. Journal of Vertebrate Paleontology 17: 137-166.

Novas, F.E.; Agnolin, F.L. 2004. Unquillosaurus ceibali 
Powell, a giant maniraptoran (Dinosauria, Theropoda) from the Late Cretaceous of Argentina. Revista del Museo Argentino de Ciencias Naturales, Nueva Serie 6 (1): 61-66.

Novas, F.E.; Bandyopadhyay, S. 2001. Abelisaurid pedal unguals from the Late Cretaceous of India. In International Symposium on Mesozoic Terrestrial Ecosystems, No. 7, Asociación Paleontológica Argentina, Publicación Especial 7: 145-149. Buenos Aires.

Novas, F.E.; Pol, D. 2002. Alvarezsaurid relationships reconsidered. In Mesozoic Birds: Above the Head of Dinosaurs (Chiappe, L.M.; Witmer, L.M.; editors). University of California Press: 121-125. Berkeley.

Novas, F.E.; Canale, J.I.; Isasi, M.P. 2003. Un terópodo maniraptor del Campaniano-Maastrichtiano del norte patagónico. Ameghiniana 40 (4): suplemento 63R.

Perle, A.; Norell, M.A.; Chiappe, L.M.; Clark, J.M. 1993. Flightless birds from the Cretaceous of Mongolia. Nature 362: 623-626.

Perle, A.; Chiappe, L.A.; Barsbold, R.; Clark, J.M.; Norell, M.A. 1994. Skeletal morphology of Mononykus olecranus (Theropoda: Avialae) from the Late Cretaceous of Mongolia. American Museum Novitates 3105: 1-29.

Powell, J.E. 1979. Sobre una asociación de dinosaurios y otras evidencias de vertebrados del Cretácico Superior de la región de la Candelaria, Provincia de Salta, Argentina. Ameghiniana 16: 191-204.

Powell, J.E. 1992. Hallazgo de huevos asignables a dinosaurios titanosáuridos (Saurischia, Sauropoda) de la Provincia de Río Negro, Argentina. Acta Zoológica Lilloana 41: 381-389.

Salgado, L.; Azpilicueta, C. 2000. Un nuevo saltasaurino (Sauropoda, Titanosauridae) de la provincia de Río
Negro (Formación Allen, Cretácico Superior), Patagonia, Argentina. Ameghiniana 37: 259-264.

Salgado, L.; Coria, R.A. 1996. First evidence of an ankylosaur (Dinosauria, Ornithischia) in South America. Ameghiniana 33: 367-371.

Salgado, L.; Parras, A.; Gasparini, Z. 2007a. Un plesiosaurio de cuello corto (Plesiosauroidea, Polycotylidae) del Cretácico Superior del norte de Patagonia. Ameghiniana 44: 349-358.

Salgado, L.; Coria, R.A.; Magalhães Ribeiro, C.; Garrido, A.; Rogers, R.; Simón, M.E.; Arcucci, A.B.; Curry Rogers, K.; Carabajal, A.P.; Apesteguía, S.; Fernández, M.; García, R.; Talevi, M. 2007b. Upper Cretaceous dinosaur nesting sites of Río Negro (Salitral Ojo de Agua and Salinas de Trapalcó-Salitral de Santa Rosa), northern Patagonia, Argentina. Cretaceous Research 28: 392-404.

Suzuki, S.; Chiappe, L.M.; Dyke, G.J.; Watabe, M.; Barsbold, R.; Tsogtbaatar, K. 2002. A new specimen of Shuvuuia deserti Chiappe et al., 1998 from the Mongolian Late Cretaceous with a discussion of the relationships of alvarezsaurids to other theropod dinosaurs. Contribution in Science 494: 1-18.

Uliana, M.A.; Biddle, K.T. 1988. Mesozoic-Cenozoic paleogeographic and geodynamic evolution of Southern South America. Revista Brasileira de GeoCiências 18: $172-190$.

Wilson, J.A. 1999. A nomenclature for vertebral laminae in sauropods and other saurischian dinosaurs. Journal of Vertebrate Paleontology 19: 639-653.

Zhao, Z. 1975. Microstructure of the dinosaurian eggshells of Nanxiong, Guangdong, and the problems in dinosaur egg classification. Vertebrata PalAsiatica 13: $105-117$. 